\title{
Zur Struktur der Solenocyten (Cyrtocyten) von Anaitides mucosa (Annelida, Polychaeta)
}

\author{
K. Hausmann \\ Lehrstuhl für Zellenlehre der Universität Heidelberg; \\ Im Neuenheimer Feld 230, \\ D-6900 Heidelberg, Bundesrepublik Deutschland
}

\begin{abstract}
On the structure of the solenocytes (cyrtocytes) of Anaitides mucosa (Annelida, Polychaeta). Based on electron microscopic observations, the structure of the solenocytes of $A$. mucosa is described. The tube of the solenocyte is made up of $14-15$ rods. These rods, which are filled with regularly packed filaments, are interconnected by an amorphous to filamentous substance. A single flagellum, lying in the tube, is surrounded by a sheet of amorphous material. The functional organization of the solenocytes is discussed.
\end{abstract}

\section{EINLEITUNG}

Die exkretorischen Organe verschiedener tierischer Organismen sind morphologisch und physiologisch eingehend untersucht worden (vgl. Kümmel, 1977), und speziell zu den Protonephridien liegen zahlreiche Untersuchungen vor (vgl. Brandenburg, 1966, 1975; Kümmel, 1975; Wilson \& Webster 1974). Ungeachtet der Fülle von Informationen sind die ultrastrukturellen Daten über die bei vielen Polychaeten ausgebildeten Solenocyten jedoch sehr spärlich. In dieser Arbeit wird daher über die Feinstruktur der Solenocyten des Phyllodociden Anaitides mucosa berichtet.

\section{MATERIAL UND METHODEN}

Die Untersuchungsorganismen wurden im Norderneyer Watt gesammelt. Die Objekte wurden für die Elektronenmikroskopie nach Franke et al. (1969) fixiert und in Epon 812 eingebettet. Die Ultradünnschnitte wurden mit einem Philips EM 400 untersucht.

\section{ERGEBNISSE UND DISKUSSION}

Die blind im Coelom endenden Protonephridien sind bei den Phyllodociden mit Terminalzellen, speziell mit Solenocyten, ausgestattet (Fig. 1a). Die Solenocyten werden in einen Terminalabschnitt, der den Kern beinhaltet, und einen röhrenförmigen Fortsatz, in dem eine Geißel schwingt, unterteilt (Fig. 1b).

Solenocyten, die im Bereich des Röhrenfortsatzes geschnitten sind, erinnern in ihrem Aufbau an die Kragenstruktur von Choanoflagellaten und an Coelenteraten- 

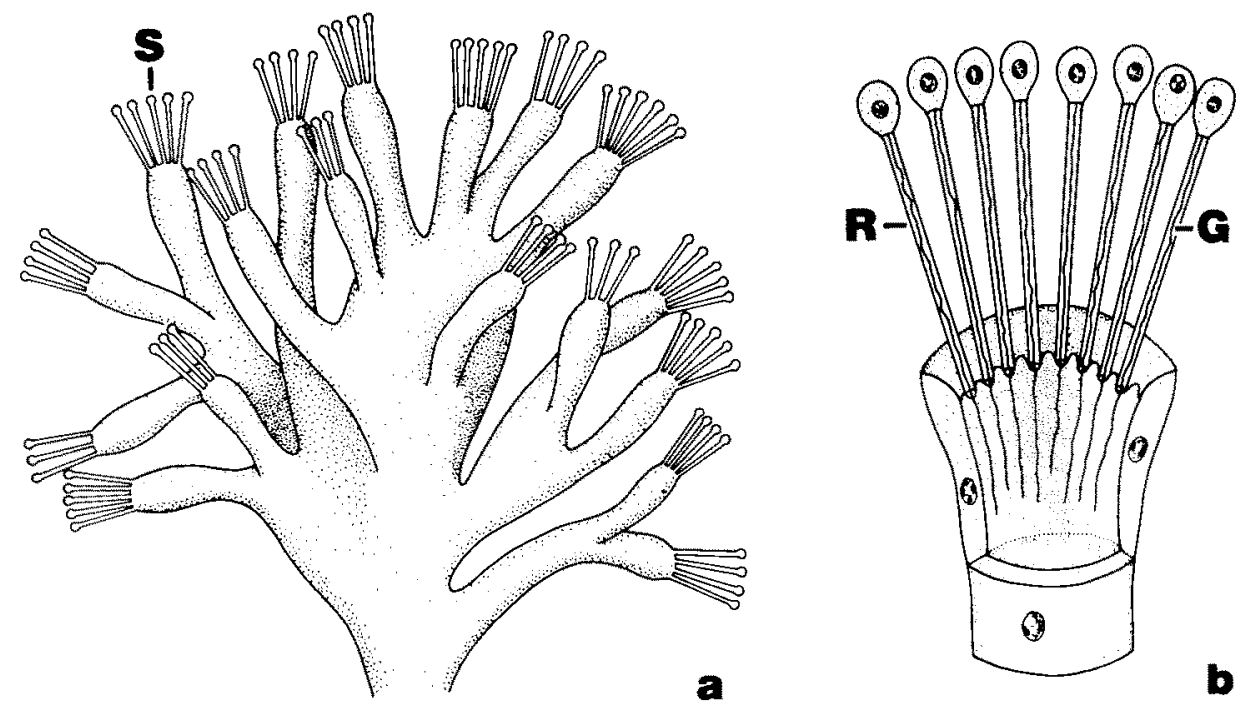

Fig. 1. Schematische Darstellung eines Polychaeten-Protonephridiums mit Solenocyten (nach Goodrich, 1945). (a) Ubersicht, (b) Ausschnitt aus einer Endigung mit Solenocyten. G-Geißel; R-Reuse; S-Solenocyte

Kragengeißelzellen. Bei Anaitides mucosa finden sich 14-15 Elemente, welche die reusenartig durchbrochene Wandung der Röhre bilden (Fig. 2a und b). Im Lumen der Röhre erkennt man das Flagellum, das in den elektronenmikroskopischen Abbildungen zum einen zentrisch, zum anderen exzentrisch angeordnet ist. Die verschiedenen Anordnungen der Geißel in der Röhre spiegeln das Schlagen dieses Organells wider.

Die Einzelelemente der Röhrenwandung, die auf Grund der im Querschnitt regelmäßig angeordneten Filamente die Struktur von Mikrovilli zeigen, sind untereinander durch eine $30 \mathrm{~nm}$ dicke Schicht amorphen, möglicherweise filamentösen Materials verbunden. Schnitte durch unterschiedliche Bereiche der Solenocyten belegen, daß das verbindende Material bis hin in die Zellareale mit dem Zellkern reicht. Darüber hinaus finden sich insbesondere in der Nähe des Terminalabschnittes filamentöse Verbindungen zwischen Wandung und zentralem Flagellum. Das Flagellum ist in diesern Bereich von einer Schicht dichten Materials umgeben. Weiter innerhalb der Terminalregion setzt sich dieses Material ringförmig vom Flagellum ab (Fig. 3).

Die Solenocyten werden auf Grund elektronenmikroskopischer Untersuchungen auch als Cyrtomastigocyten (Reusengeißelzellen), oder kurz Cyrtocyten, bezeichnet (Kümmel \& Brandenburg, 1961). Die "Reuse" ist ein funktionell wichtiger Bereich der Solenocyte. Denn hier kann die Körperflüssigkeit ins Innere des röhrenförmigen Abschnittes dringen. Brandenburg (1966) vermutete, daß diese Zellabschnitte zur Filtration befähigt sind, da die Schlitze durch, wie er beobachtete, membranartige Strukturelemente verschlossen werden. Wilson \& Webster (1974) dagegen stellen in Zweifel, ob ein einzelnes Flagellum einen genügend großen hydrostatischen Druckgradienten erzeugen kann, der für eine echte Ultrafiltration ausreichen würde. Diese Autoren 


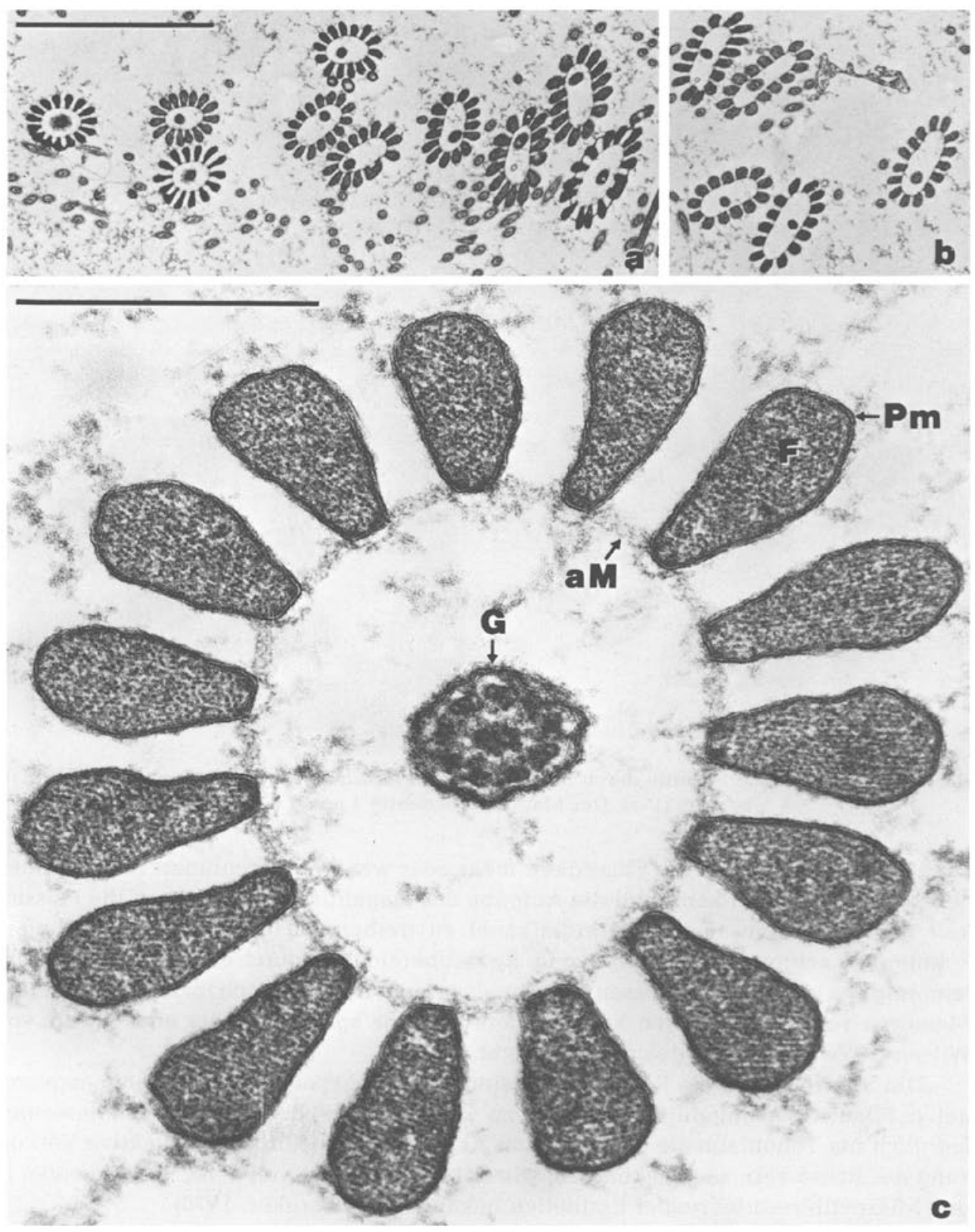

Fig. 2. Querschnitte durch den Reusenbereich der Solenocyton von Anaitides mucosa. (a) Ubersicht; x 5000; (b) Starke Vergrößerung, aM = amorphes Material; $\mathrm{F}=$ Filamente; $\mathrm{G}=\mathrm{Geißel}$; Pm = Plasmamembran; $\times 80000$. Der Maßstrich in (a) gibt 5 um an, in (b) $0,5 \mu m$ 


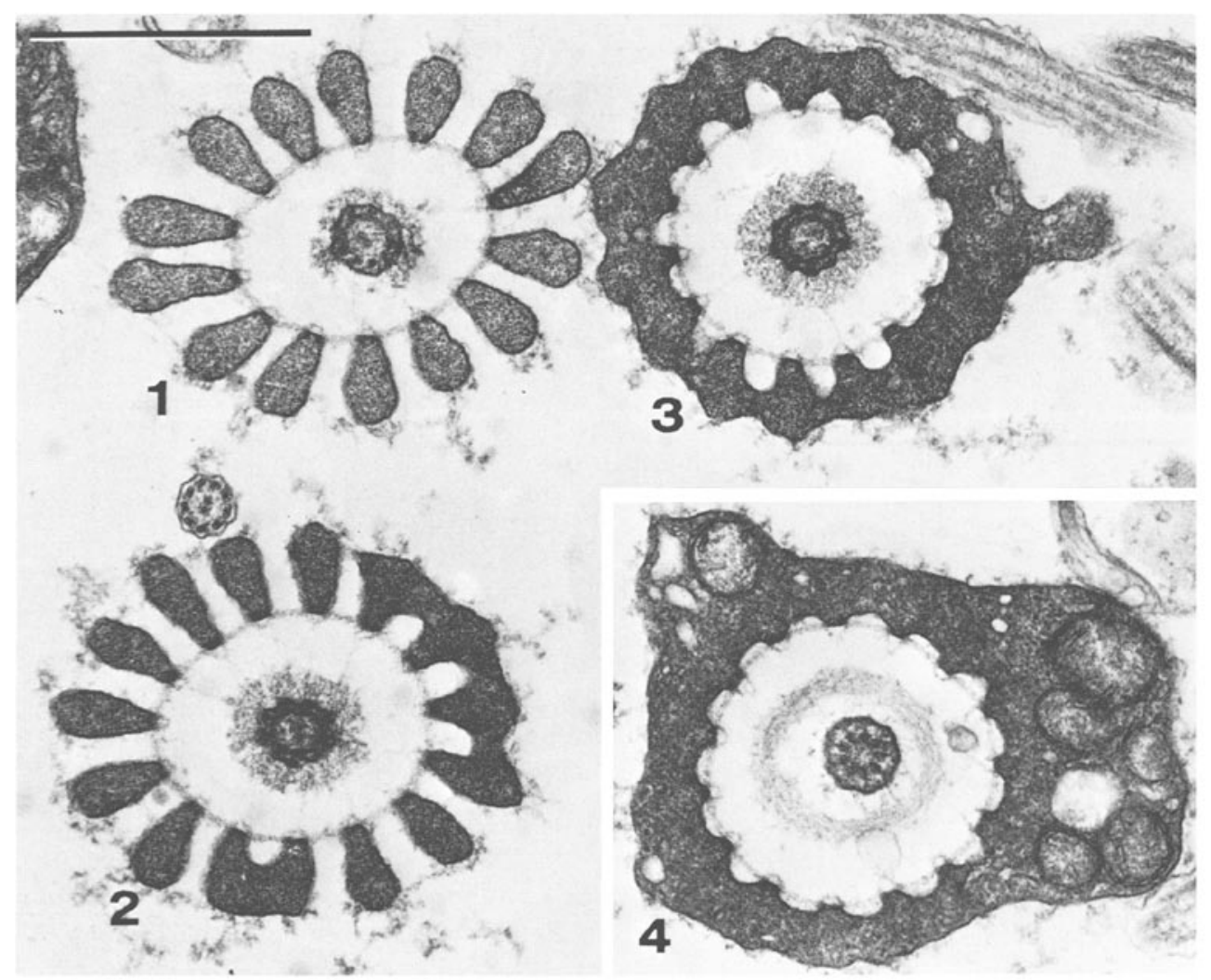

Fig. 3. Unterschiedliche Schnilte durch Solenocyten von Anailides mucosa in Richtung Terminalbereich (1-4). Der Maßstrich bedeutet 1 um; x 30000

vermuten vielmehr, daß die Flüssigkeit mehr oder weniger ungehindert in das Lumen der Röhre eindringen kann und die Aufgabe des Flagellums darin bestehı, die Flüssigkeit in den gemeinsamen Nephridialkand zu treiben. In unseren Untersuchungen konnten wir zeigen, daß die Schlitze im Reusenbereich nur durch ein lockeres, amorphfeinlädiges Malerial verschlossen werden, das weder in Dicke noch im Aufbau mit einer Membran verglichen werden kann. Unsere Befunde sprechen daher eher für die von Wilson \& Webster (1974) geäußerte Ansicht.

Die Stäbe, welche die Reuse bilden, sind mit dichtgepackten, regelmäßig angeordneten Filamenten angefüllt. Es bleibt zu klären, ob es sich bei diesen Filamenten lediglich um Tonofilamente oder aber um Aktinfäden handelt, die eine aktive Verkürzung der Reuse verursachen könnten, wie es beispielsweise von den Corefilamenten in den Mikrovilli resorbierender Epithelien bckannt ist (Moosckor, 1976).

Danksagungen. Für die Hilte bei der Materialbeschaffung danke ich Hern Dr. H. Michaelis, Forschungsstelle für Insel-und Küstenschutz, Vordeney. Hemu Prof. Dr. G. Kümmel, Universitat Karlsruhe, danke ich für wertvolle Literaturhinweise. Umsichtige technische Assistenz leistete Frl. A. Rüskens. 


\section{ZITIERTE LITERATUR}

Brandenburg, $J_{2}$ 1966. Die Reusenformen der Cyrtocyten. - Zool. Beitr. 12, 345-417.

Brandenburg, J., 1975. The morphology of the protonephridia. - Fortschr. Zool. 23, 1-17.

Franke, W. W., Krien, S. \& Brown R. M. jr., 1969. Simultaneous glutaraldehyde-osmium tetroxide fixation with postosmication. An improved fixation procedure for electron microscopy of plant and animal cells. - Histochemie 19, 162-164.

Goodrich, E. S., 1945. The study of nephridia and genital ducts since 1895. - Q. Jl. microsc. Sci. 86, 113-393.

Kümmel, G., 1975. The physiology of protonephridia. - Fortschr. Zool. 23, 18-32.

Kümmel, G., 1977. Der gegenwärtige Stand der Forschung zur Funktionsmorphologie exkretorischer Systeme, Versuch einer vergleichenden Darstellung, - Verh. dt. Ges. Zool. 70, 154-174.

Kümmel, G. \& Brandenburg, J., 1961. Die Reusengeißelzellen (Cyrtocyten).-Z. Naturf. 16b, 692-697.

Mooseker, M. S., 1976. Actin filament-membrane attachment in the microvilli of intestinal epithelial cells. In: Cell motility. B: Actin, myosin and associated proteins. Ed. by R. Goldman, T. Pollard \& J. Rosenbaum. Cold Spring Harbor Laboratory, 631-650.

Wilson, R. A. \& Webster, L. A., 1974. Protonephridia. - Biol. Rev. 49, 127-160. 